\title{
Comparison of arterial waves derived by classical wave separation and wave intensity analysis in a model of aortic coarctation
}

\author{
Jeroen P. H. M. van den Wijngaard • \\ Maria Siebes $\cdot$ Berend E. Westerhof
}

Received: 16 December 2007/ Accepted: 15 August 2008/Published online: 2 September 2008

(C) The Author(s) 2008. This article is published with open access at Springerlink.com

\begin{abstract}
Coarctation of the aorta may develop during fetal life and impair quality of life in the adult because upper body hypertension and aneurysm formation in the descending aorta may develop. We used our computational model of the young adult arterial circulation, incorporated aorta coarctation over a range from 0 to $80 \%$ and evaluated the effects in terms of forward pressure $\left(P^{+}\right)$and backward pressure $\left(P^{-}\right)$. Predictions at several sites proximal and distal to the coarctation using an impedance-based waveform separation method (WSA) and the time-domain technique of wave intensity analysis (WIA) yielded comparable outcomes. A large reflected backward compression wave was seen proximal to the coarctation. Both techniques, WSA and WIA, gave the same results in terms of $P^{+}$and $P^{-}$. A descending index (DI) was formulated as the difference between peak systolic pressure and valve closure pressure, divided by the pulse pressure. DI increased with stenosis severity for mild to moderate aortic coarctations that did not yet cause evident hypertension. This index may allow for early diagnosis by noninvasive estimation of coarctation severity.
\end{abstract}

Keywords Impedance analysis .

Wave intensity analysis - Systemic circulation .

Windkessel $\cdot$ Aortic stenosis

J. P. H. M. van den Wijngaard $(\bowtie) \cdot$ M. Siebes

Department of Medical Physics, Academic Medical Center,

University of Amsterdam, Meibergdreef 9,

1105 AZ Amsterdam, The Netherlands

e-mail: j.p.vandenwijngaard@amc.uva.nl

B. E. Westerhof

BMEYE Cardiovascular Monitoring, Academic Medical Center,

University of Amsterdam, Amsterdam, The Netherlands

\section{Introduction}

Coarctation of the aorta is an important malformation which may occur during fetal development. When developing in isolation, this anomaly may be difficult to detect antenatally. In a number of cases, aortic coarctation may go unnoticed until signs of the development of upper body hypertension and a weak and delayed femoral pulse in young adults. In more severe cases, this may develop into left ventricular hypertrophy [15] and dilatation and outwards remodeling of collateral arteries from the intercostal arteries that branch back into the descending aorta. Treatment of aortic coarctation includes excision of the constricted segment and the subsequent creation of an end-to-end anastomosis, placement of a synthetic patch, or balloon dilatation and stenting [1]. The most important predictor of successful long-term outcome is age at time of initial repair [4], and thus early detection of coarctation.

To investigate the influence of the aortic coarctation on the reflected pressure waves traveling towards the heart, a model of the adult human circulation was developed. Two techniques for evaluation of reflected waves were applied. The first method will be referred to as the wave separation (WSA) method, where pressure is separated into its forward and backward traveling components as proposed by Westerhof et al. [25]. In the second method, referred to as wave intensity analysis (WIA), originally introduced by Parker and Jones $[16,17]$, a measure of the energy of the reflected wave that returns towards the heart is obtained. We used an existing model of the human circulation to compare the results of both methods. From our results, we propose a pressure-based parameter as measure of aortic coarctation severity. 


\section{Methods}

\subsection{Distributed model}

For the development of a segmented model of the arterial circulation of the adult, the previous model of Westerhof et al. [22, 23] was used and extended using data of Stergiopulos et al. [18]. These data have formed the basis for previous studies of wave propagation, for example by Wang and Parker [21].

The distributed model consists of arterial segments, each with its length and diameter, giving longitudinal impedance $Z_{L}$ per unit length using Womersley's oscillatory flow theory, and the radius, wall thickness, wall viscoelasticity, and Young's modulus as determined previously [18], giving transverse impedance $Z_{T}$ per unit length. The various distal organ beds were simulated by three-element Windkessels [24], i.e. by two resistances and a compliance, known to represent organ beds well. In total, the model contained 121 arterial segments and 30 three-element Windkessels. Details on the distributed model can be found elsewhere [18, 22]. Propagation of a pressure and flow pulse through the arterial tree was calculated firstly by decomposing the flow and pressure waves into frequency components by standard Fourier analysis. The propagation of each frequency harmonic through the arterial tree can be computed by transfer function methodology. All attenuated and phase shifted frequency components can be recomposed into the time domain by inverse Fourier analysis to give pressure and flow in the time domain. A flow waveform, measured in the human ascending aorta, [12] is used as input flow. The basic equations pertaining to frequency domain analysis approach are briefly outlines here. Details can be found elsewhere [11, 24, 25].

The transfer function describing pressure $\left(H(\omega)_{\text {Pressure }}\right)$ as a function of frequency $\omega$ can be defined as follows

$H(\omega)_{\text {Pressure }}=\frac{P(\omega)_{\text {Distal }}}{P(\omega)_{\text {Proximal }}}$

For clarity however in Eq. 2 through 10 the dependence on the frequency $\omega$ has been omitted. The transfer of flow can be described similarly [28]. In the frequency domain, flow and pressure are related through the characteristic impedance. From the longitudinal $\left(Z_{L}\right)$ and transverse $\left(Z_{T}\right)$ impedances of each vessel segment, the characteristic impedance $\left(Z_{0}\right)$ and propagation coefficient $\gamma$ follow as

$Z_{0}=\sqrt{Z_{L} Z_{T}}$

$\gamma=\sqrt{Z_{L} / Z_{T}}$

The propagation coefficient, $\gamma$, consists of a real part, describing the damping of waves due to viscous friction, and an imaginary part, describing the phase delay. These parameters are calculated from segment to segment.

Using the definition of the reflection coefficient $\Gamma$ and with $Z_{\text {Load }}$ the loading impedance of the segment, i.e. the downstream impedance of the vascular tree connected at the terminus of the segment, the reflection is given as

$\Gamma=\frac{P^{-}}{P^{+}}=\frac{Z_{\text {Load }}-Z_{0}}{Z_{\text {Load }}+Z_{0}}$

The propagation of forward and backward traveling pressure, denoted by the superscripts ${ }^{+}$and ${ }^{-}$, depends on the propagation coefficient as

$P_{\text {Proximal }}^{+}=P_{\text {Distal }}^{+} \exp (\gamma l)$

$P_{\text {Proximal }}^{-}=P_{\text {Distal }}^{-} \exp (-\gamma l)$

with $l$ representing segment length. The transfer function of pressure can be derived [28] as:

$H_{\text {Pressure }}=\frac{1+\Gamma}{1+\Gamma \mathrm{e}^{-2 \gamma l}} \mathrm{e}^{-\gamma l}$

The transfer function of flow can be derived similarly

$H_{\text {Flow }}=\frac{1-\Gamma}{1-\Gamma \mathrm{e}^{-2 \gamma l}} \mathrm{e}^{-\gamma l}$

The input impedance of segments in series follows as

$Z_{\text {IN }}=\frac{H_{\text {Flow }}}{H_{\text {Pressure }}} Z_{\text {Load }}$

\subsection{Wave separation}

Separation of the measured pressure or flow wave into the forward and backward traveling waves is done according to Westerhof [25]. In brief, since forward and backward waves are related through the reflection coefficient in Eq. 4, the forward and backward pressure can be described as

$P^{+}=\frac{P}{1+\Gamma}$

$P^{-}=\frac{P \Gamma}{1+\Gamma}$

Since the reflection coefficient can also be represented in terms of the characteristic and the load impedances, Eq. 4, and the characteristic impedance is the ratio of the measured flow and pressure, the forward and backward pressure waves can be obtained as

$P^{+}=\left(P+F Z_{0}\right) / 2$

$P^{-}=\left(P-F Z_{0}\right) / 2$

The relation for forward and backward flow can be derived similarly 
$F^{+}=\left(F+P / Z_{0}\right) / 2$

$F^{-}=\left(F-P / Z_{0}\right) / 2$

\subsection{Analysis of wave intensity}

Wave intensity analysis (WIA) was introduced by Parker and Jones $[16,17]$ as a time domain method. This method is especially useful for the study of non-periodic transient flow and flows where the load impedance of the system is varying strongly, e.g. during a heart cycle in the coronary arterial system [5, 20, 29]. Pressure and flow waves are assumed to travel in the forward direction with the sum of the wave speed, $c$, and the blood flow velocity, $U$, whereas the backward traveling waves are assumed to travel with the difference of the wave speed, and blood flow velocity, in the backward direction.

Assuming conservation of mass and negligible viscous losses, Parker et al. [16] derived that the forward and backward components of the measured pressure and flow velocity $(U)$ waves in elastic tubes can be described by

$$
\begin{aligned}
& \mathrm{d} P^{+}=(\mathrm{d} P+\rho c \mathrm{~d} U) / 2 \\
& \mathrm{~d} P^{-}=(\mathrm{d} P-\rho c \mathrm{~d} U) / 2 \\
& \mathrm{~d} U^{+}=(\mathrm{d} U+\mathrm{d} P / \rho c) / 2 \\
& \mathrm{~d} U^{-}=(\mathrm{d} U-\mathrm{d} P / \rho c) / 2
\end{aligned}
$$

where $\rho$ is the blood density and $c$ is the wave speed, which follows from the segment properties using a modified Moens-Korteweg relation. In our case, wave speed in the aortic arch typically was about $3.6 \mathrm{~m} \mathrm{~s}^{-1}$.

In WIA the macroscopic waves are considered to be the superposition of infinitesimal discrete wavefronts, manifested by changes in $P$ and $U$. The separated wave intensity, expressed as the rate of energy flux per unit area, $\mathrm{W} \mathrm{m}^{-2}$, can be calculated from the product of $\mathrm{d} P^{+}$and $\mathrm{d} U^{+}$ describing the intensity of the forward traveling wave and $\mathrm{d} P^{-} \mathrm{d} U^{-}$as the intensity of the backward traveling wave [20].

$$
\begin{aligned}
& \mathrm{d} I^{+}=\mathrm{d} P^{+} \mathrm{d} U^{+}=(\mathrm{d} P+\rho c \mathrm{~d} U)^{2} / 4 \rho c \\
& \mathrm{~d} I^{-}=\mathrm{d} P^{-} \mathrm{d} U^{-}=-(\mathrm{d} P-\rho c \mathrm{~d} U)^{2} / 4 \rho c
\end{aligned}
$$

In our computations, instead of obtaining $\mathrm{d} P$ and $\mathrm{d} U$ as the difference between sequential time intervals, we used the time derivatives of $P$ and $U$ to seconds $s$, resulting in normalized wave intensity expressed as $\mathrm{W} /\left(\mathrm{m}^{2} \mathrm{~s}^{2}\right)$. The sum of the forward and backward wave intensities yields the net wave intensity as $\mathrm{d} I=\mathrm{d} P \mathrm{~d} U$.

In our model simulation, $d I$ was obtained using WIA as well as wave separation [25]. In WIA, four different types of waves can be distinguished, i.e., Forward Compression Wave, FCW, where $\mathrm{d} P^{+}>0$; Backward Compression Wave, BCW, where $\mathrm{d} P^{-}>0$, Forward Expansion Wave, FEW, where $\mathrm{d} P^{+}<0$ and the Backward Expansion Wave,
BEW, where $\mathrm{d} P^{-}<0$. An in-depth overview of WIA and its application is provided by Jones $[8,9]$.

Differences between WSA and WIA for the separated pressure and velocity waves and for the wave intensities were described by calculating the root mean square deviation (RMSD), normalized by the average of the absolute values during the heart cycle.

\subsection{Simulations of normal and coarctation conditions}

Simulations were first performed for the normal aorta, which was used as a reference, assuming a normal internal radius of $1 \mathrm{~cm}$. Subsequently, aortic coarctation was modeled directly beyond the brachiocephalic trunk and the left subclavian artery, at $9.9 \mathrm{~cm}$ from the aortic valve. A series of coarctation severities ranging from 0 up to $80 \%$ diameter stenosis, i.e. an area stenosis of $96 \%$, was simulated by reducing the diameter of the first thoracic aorta segment, measuring $5.2 \mathrm{~cm}$ in length, from 0 to $80 \%$ in steps of $20 \%$. In addition, we increased the wall stiffness by doubling Young's modulus from 2 to $4 \times 10^{5} \mathrm{~Pa}$. Both the maximal severity of the luminal narrowing and the increase in aorta stiffness were within recorded limits of aortic coarctation in humans $[15,19]$. Heart rate was modeled as constant, at 73 beats per minute, and cardiac output was kept unchanged.

Blood pressure was calculated at three locations proximal to the stenosis (distal aortic arch, carotid artery and radial artery) and in the femoral artery, distal to the stenosis. From these, mean arterial pressure, MAP, pulse pressure, PP, as well as forward and backward traveling pressure waves were derived. The pressure waves for the normal and coarctation condition were compared at the level of the distal aortic arch. Finally, we defined a descending index (DI) as the difference between the peak systolic pressure (PSP) and the pressure at aortic valve closure (PAV), taken as the pressure at the time of the incisura, divided by the pulse pressure

$\mathrm{DI}=(\mathrm{PSP}-\mathrm{PAV}) / \mathrm{PP}$

This DI was evaluated for a range of aortic coarctation up to $80 \%$.

\section{Results}

Results are shown for the normal aorta and the $80 \%$ diameter coarctation, showing the maximal change from the control situation.

\subsection{Arterial input impedance}

Input impedance in terms of modulus and phase of the arterial circulation is shown in Fig. 1 for the reference condition and for an $80 \%$ aortic coarctation. Total arterial 

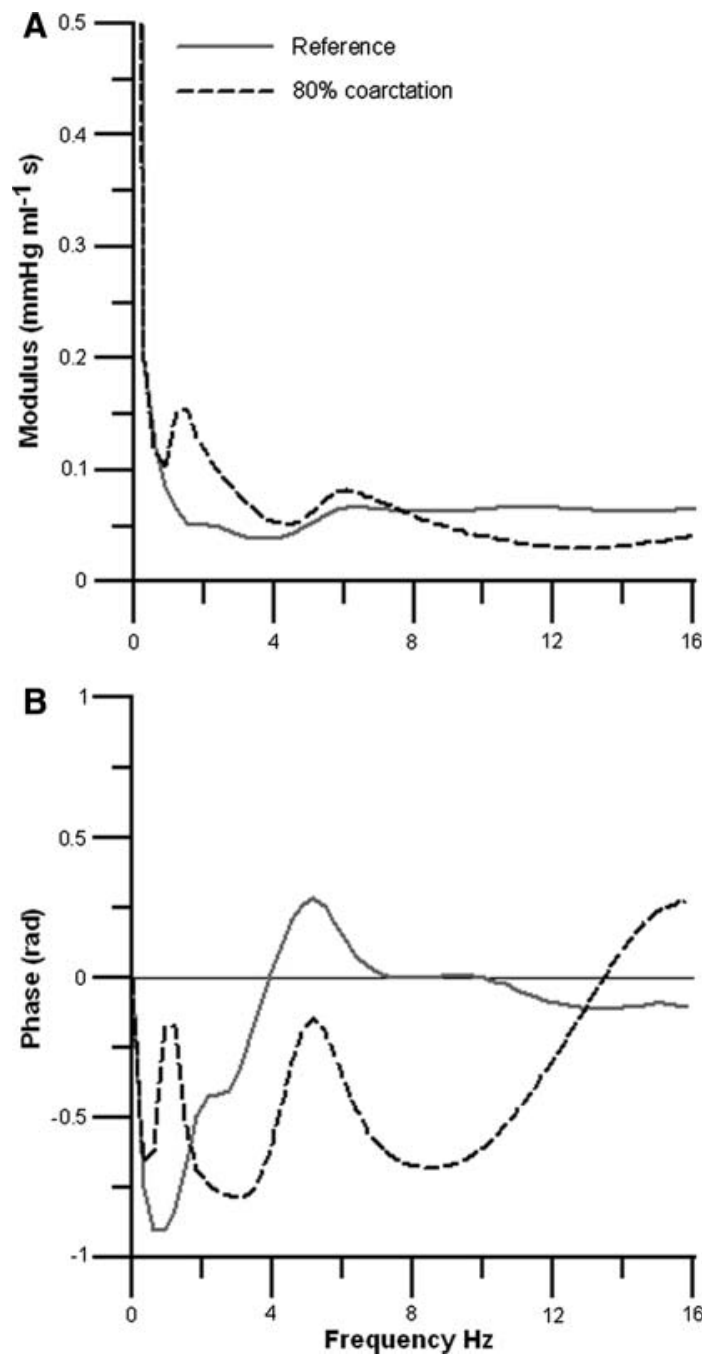

Fig. 1 Magnitude (a) and phase (b) of the arterial input impedance in the normal aorta and with $80 \%$ diameter coarctation. The impedance at $0 \mathrm{~Hz}$, i.e. the resistance, was $1.11 \mathrm{mmHg} \mathrm{ml}^{-1} \mathrm{~s}$ in the normal case, and $1.23 \mathrm{mmHg} \mathrm{ml}^{-1} \mathrm{~s}$ in the coarctation condition

resistance increased from 1.11 for the normal aorta to $1.23 \mathrm{mmHg} \mathrm{ml}{ }^{-1} \mathrm{~s}$ in the $80 \%$ coarctation case. The phase angle was predominantly negative and decreased with aortic coarctation (Fig. 1b).

\subsection{Aortic arch, carotid, radial, and femoral artery pressures}

Arterial pressures for the reference case in the distal aortic arch, carotid, radial and femoral artery are shown in Fig. 2a. Pulse pressure was elevated in the proximal and peripheral arteries, and the time of the peak pressure was delayed compared to the peak in the aortic arch.

For the $80 \%$ diameter coarctation, the pulse, mean, and PSPs at all proximal locations increased (Fig. 2b). A summary of representative pressures is shown in Table 1 .
In our model simulation however, this simulation of severe coarctation did not give rise to evident hypertension, i.e. the systolic/diastolic blood pressure increased from 114/79 to $146 / 94 \mathrm{mmHg}$ in the aortic arch and from 123/75 to $162 /$ $93 \mathrm{mmHg}$ in the radial artery. The moderate increase in pressure here is both a consequence of the unaffected model elements in parallel connection, i.e. the right and left arm and carotid arteries [23], and the remaining lumen of the coarctation element. Compared to the normal condition, PSP with the $80 \%$ diameter coarctation was slightly delayed by about $0.01 \mathrm{~s}$ for the proximal locations, whereas in the distal femoral artery, PSP arrived at $0.1 \mathrm{~s}$ later than in the normal condition.

3.3 Comparing forward and backward pressure waves in the aortic arch

The simulated pressure in the aortic arch and respective forward and backward traveling pressure waves are compared in Fig. 3 for the normal condition (black lines) and the $80 \%$ diameter coarctation case (gray lines). Figure $3 a$ illustrates results obtained with WSA, while Fig. $3 b$ shows the results obtained by WIA.

Compared to the reference case, both methods show that the magnitude of the backward pressure wave in aortic coarctation was markedly augmented in systole, leading to a substantial rise in the total pressure. Forward and backward pressure waves started to rise approximately simultaneously.

\subsection{Comparing wave separation and wave intensity analysis in the reference condition}

Separated pressure and flow velocity waves at the level of the aortic arch obtained by WIA are shown in Fig. $4 a$ and $b$ for the reference condition. In the initial 0.1 seconds a large increase in pressure and velocity is generated by the ventricle, causing a forward compression wave as shown in Fig. 4c. The subsequent decrease in pressure and velocity gives rise to a forward traveling expansion wave in the time interval from 0.2 to $0.3 \mathrm{~s}$. Wave separation analysis yielded comparable results to WIA (not shown). Differences between WIA and WSA were quantified by calculating the RMSD of each of the parameters. For the separated pressure waves, $\mathrm{d} P^{+}$and $\mathrm{d} P^{-}$, a normalized RMSD of 36 and $9 \%$ was found and for the separated velocity waves, $\mathrm{d} U^{+}$ and $\mathrm{d} U^{-}, 39$ and $128 \%$ was found. These findings indicate that the pressure derivatives were more similar than the velocity derivatives. The resulting separated forward and backward wave intensities depicted in Fig. 4c illustrate that forward wave intensity is dominant in the normal aorta. For the forward and backward wave intensities, the normalized RMSD was 10 and $214 \%$ respectively. The difference in wave intensity was largely due to the difference in the 
Fig. 2 Arterial pressures at proximal (aortic arch, radial and carotid artery) and distal (femoral artery) locations in the reference case (left) and for a $80 \%$ diameter coarctation (right)

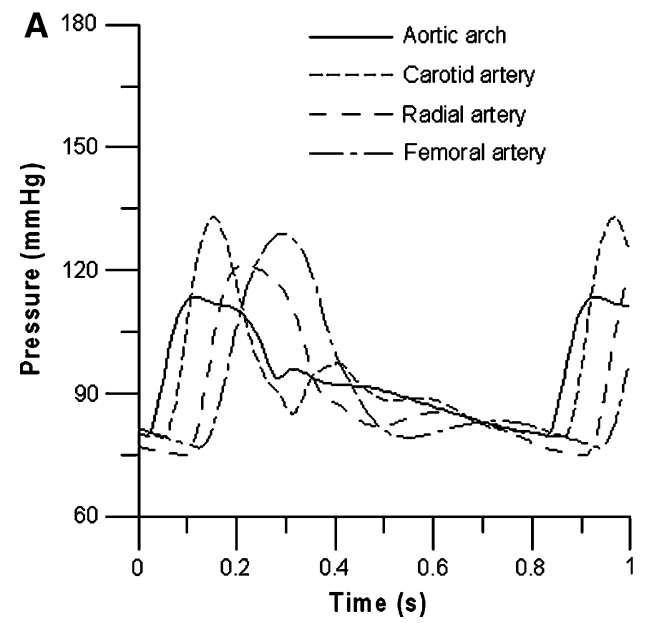

Table 1 Mean arterial pressure (MAP), pulse pressure (PP), and peak systolic pressure (PSP), (in $\mathrm{mmHg}$ ) for normal aorta and $80 \%$ diameter aortic coarctation

\begin{tabular}{llllllll}
\hline Location & \multicolumn{3}{l}{ Normal aorta } & & \multicolumn{3}{c}{$80 \%$ coarctation } \\
\cline { 2 - 3 } \cline { 8 - 9 } & MAP & PP & PSP & & MAP & PP & PSP \\
\hline Aortic arch & 93 & 33 & 114 & & 103 & 51 & 146 \\
Carotid artery & 93 & 54 & 135 & & 103 & 83 & 178 \\
Radial artery & 90 & 46 & 123 & & 100 & 69 & 162 \\
Femoral artery & 92 & 52 & 130 & & 89 & 63 & 126 \\
\hline
\end{tabular}

separated velocity waves. As can be seen from Fig. 4c, both methods predict a large forward wave intensity from 0 to $0.1 \mathrm{~s}$, resulting from a large forward compression wave, followed by a smaller forward intensity wave, resulting from a forward expansion wave in the interval from 0.2 to $0.3 \mathrm{~s}$. In addition, both methods predict a small backward traveling compression wave between 0 and $0.1 \mathrm{~s}$. The predicted results are in concordance with previous results of WIA applied to the arterial circulation [9]. WSA predicted a slightly increased forward wave and larger backward wave intensity compared to the WIA results.
3.5 Comparing wave separation and wave intensity analysis in aortic coarctation

Wave separation and WIA of pressure and flow velocity waves at the level of the aortic arch in $80 \%$ diameter aortic coarctation (smaller degrees of diameter coarctation not shown) revealed a strong increase in backward traveling pressure and velocity waves between 0 and 0.1 seconds. Figures $5 \mathrm{a}$ and $\mathrm{b}$ show results obtained by WIA Results for WSA are not shown but were similar in shape. The normalized RMSD for was 15 and $18 \%$ for the forward and backward separated pressure and 56 and $66 \%$ for the velocity waves, respectively. These results indicate that shape and magnitude of the separated pressure waves agreed closer between the two methods than those of velocity waves. For the forward and backward wave intensities, the normalized RMSD was 70 and $114 \%$ respectively.

As is shown in Fig. 5a and b, the predicted forward traveling compression wave in the first $0.1 \mathrm{~s}$ was accompanied by a virtually equal backward traveling compression wave. A similar observation can be made for the interval between 0.2 and 0.3 seconds where a forward traveling
Fig. 3 Results for WSA (left) and for WIA (right). The normal waves are shown in black, in case of $80 \%$ diameter coarctation, waves are shown in grey. Both the net and backward traveling wave were enhanced during early systole with the distal coarctation, the backward traveling wave appears to arrive earlier in coarctation
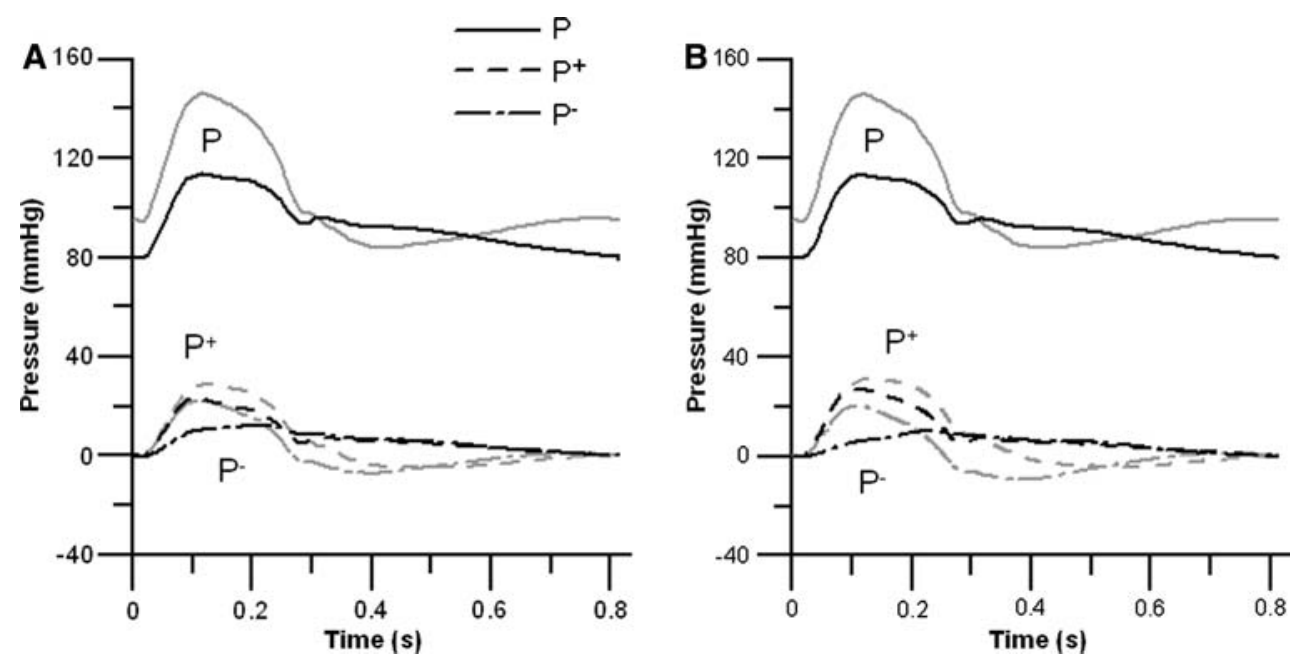

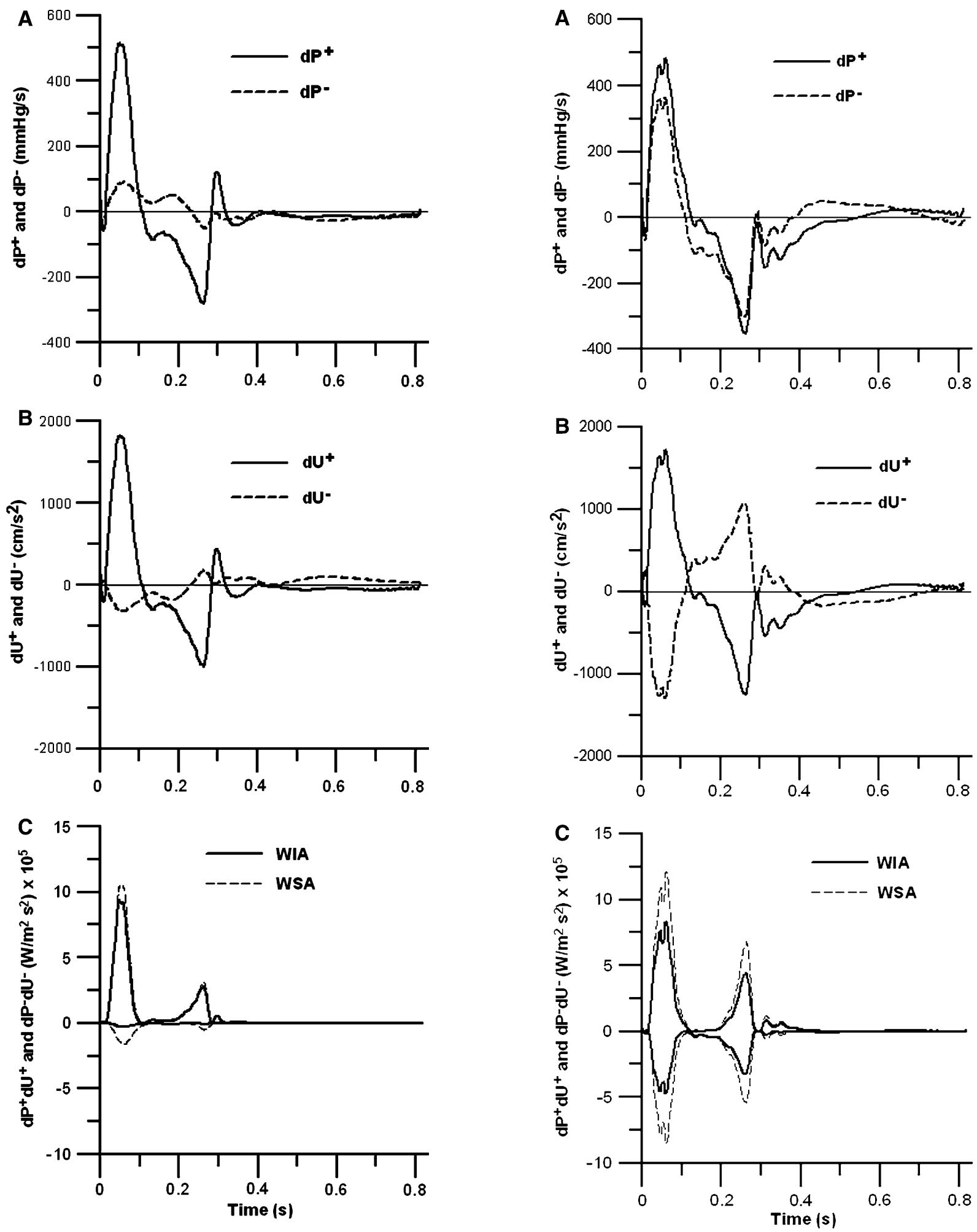

Fig. 4 Separated pressure (a) and velocity (b) waves based on WIA, for the aortic arch in the reference condition. Results obtained by wave separation were highly similar (not shown). The resulting forward and backward wave intensities were quite similar for WSA and WIA (c), with dominant forward traveling waves

Fig. 5 Separated pressure (a) and velocity (b) waves obtained by wave intensity analysis for the $80 \%$ diameter aorta coarctation. Results obtained by wave separation were highly similar (not shown). The resulting wave intensities (c) for both WSA and WIA demonstrate substantially increased backward waves compared to the reference case (Fig. 4c) 


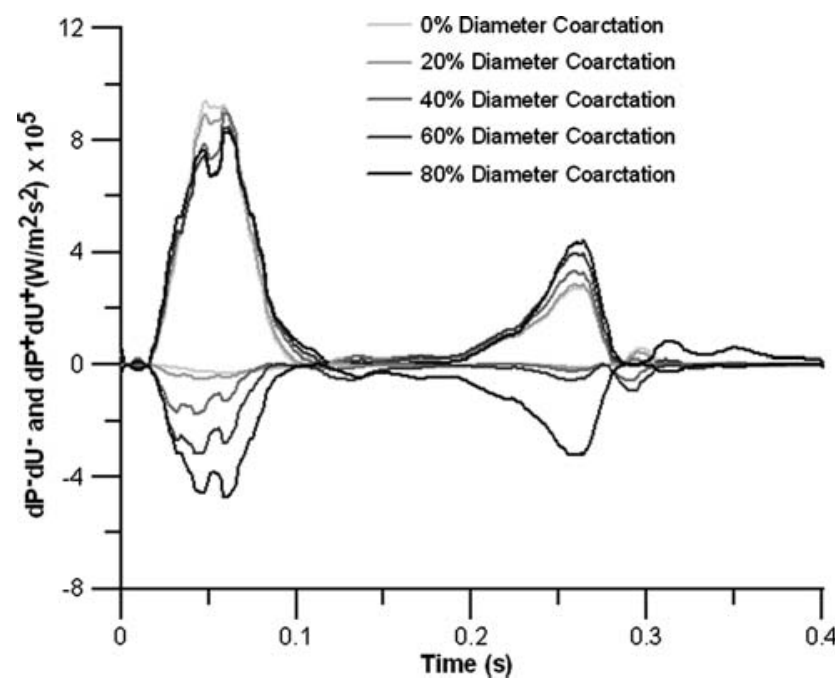

Fig. 6 Separated forward and backward wave intensities calculated at the aortic arch by WIA for different percent of diameter coarctations

expansion wave is accompanied by a backward traveling expansion wave. The resulting forward and backward wave intensities for the $80 \%$ diameter aortic coarctation (Fig. 5c) were markedly reduced compared to the reference case shown in Fig. 4c. In addition, forward and backward wave intensities were of similar in shape but opposite in sign, which was a consequence of the short distance between the site of coarctation and the modeled measurement location.

Figure 6 compares the forward and backward wave intensities obtained by WIA in aortic coarctation for coarctation severities of $0,20,40,60$ and $80 \%$. Backward wave intensity increased substantially from $0 \%$ diameter to $80 \%$ diameter stenosis, while forward wave intensity was reduced to a smaller degree by aortic coarctation. In contrast to the forward compression wave decrease, the forward traveling expansion wave between 0.2 and $0.3 \mathrm{~s}$ increased with aortic coarctation, which is a consequence of the higher pressure proximal to the coarctation, with increasing stenosis severity. In contrast to the strong increase in backward wave intensity increase between 0 and $0.1 \mathrm{~s}$, the backward wave intensity between 0.2 and $0.3 \mathrm{~s}$ increases to a much smaller degree. The time of the maximal forward and backward wave intensities was similar as a consequence of the short distance in the model between the coarctation location and the measurement location.

\subsection{Descending index}

Simulations of mild to severe aortic coarctation, i.e. 20 to $80 \%$ diameter stenosis, demonstrated that PSP at the aortic arch markedly increased for more severe coarctation, whereas the diastolic pressure increased to a much smaller

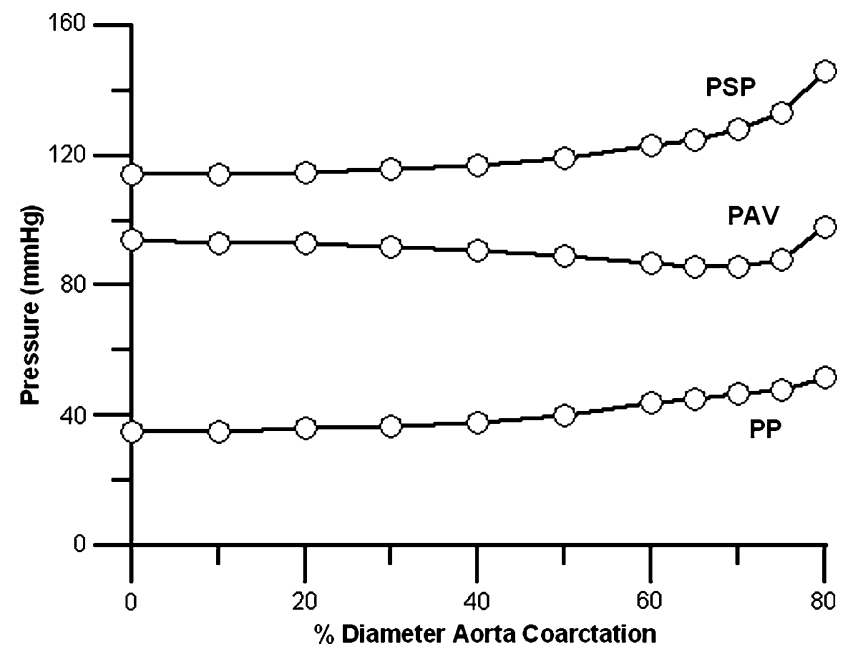

Fig. 7 Peak systolic pressure $(P S P)$, pressure at aortic valve closure $(P A V)$ and pulse pressure $(P P)$, at the aortic arch as a function of the percent diameter coarctation

degree, as shown in Fig. 7. In contrast, the pressure at valve closure decreased slightly and only started to increase above $75 \%$ diameter coarctation. The resulting DI (Eq. 13), for the aortic arch pressure in the reference case was $(111-94 \mathrm{mmHg}) /(111-79 \mathrm{mmHg})=0.53$, while for the $80 \%$ diameter coarctation DI was $(146-98 \mathrm{mmHg}) /$ $(146-96 \mathrm{mmHg})=0.96$. Figure 8 illustrates that DI increased nonlinearly with the severity of aortic coarctation. A best fit was described by the relation DI $=0.59+$ $6.4 \times 10^{-5}(\% \text { stenosis })^{2}(\mathrm{R} 2=0.99)$ not including $80 \%$

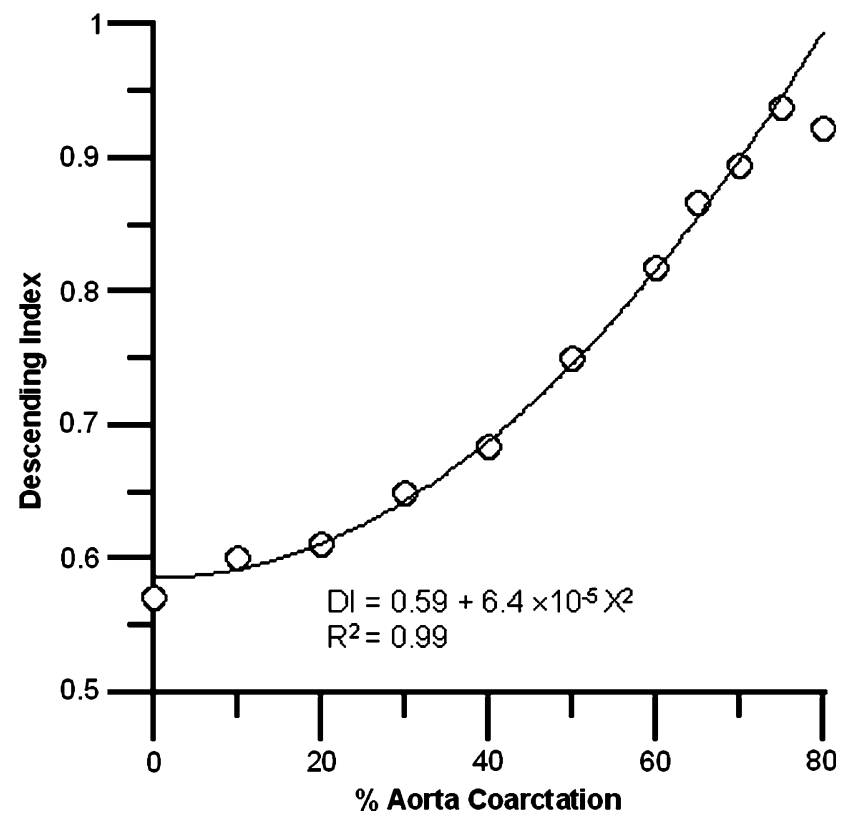

Fig. 8 Descending index $(D I)$ defined as difference between peak systolic and valve closure pressure, divided by the pulse pressure, as a function of coarctation severity 
coarctation. As can be seen in Fig. 8, DI is reduced again after $80 \%$ diameter stenosis, which is a consequence of increasing mean arterial pressure.

\section{Discussion}

In this study we compared two analysis methods; i.e. classical wave separation and WIA, in a model of aortic coarctation, to investigate the differences in the assessment of reflected waves between the two methods. Although both methods yielded similar predictions in the unobstructed reference condition as well as in the coarctation case, slight differences were found especially for the separated velocity waves.

An interesting finding of our predictions was the absence of evident hypertension, i.e. blood pressure was not substantially elevated even with moderately severe aortic coarctation. In these cases, the pressure wave developed with a steeper envelope and a concurrent increase in the difference between the PSP and the pressure at which the aortic valve closes. As a consequence, the proposed DI increased considerably with increasing severity of the coarctation. In the case simulated here, DI assessed in the aortic arch increased from 0.55 to almost 0.7 for a $40 \%$ aortic coarctation., This suggests that the use of the DI may permit early diagnosis in cases of mild coarctation and thereby improve the future quality of life for the respective patients due to early surgical intervention.

\subsection{Wave separation and wave intensity analysis}

Classical impedance analysis assumes periodicity and linearity and is based on a theory of sinusoidal wave trains that are expressed in terms of phase and frequency. For the systemic circulation at steady state, this is a reasonable assumption. In contrast, WIA is a time-based method where waves are considered the result of coincident successions of wave fronts that can be located in time and space.[3] One of the main advantages of WIA is the application of this technique in conditions where distal resistance changes transiently during the analysis period, typically the cardiac cycle. For example, the technique of WIA has proven especially useful in the coronary circulation [20]. However, in a time-invariant system, similar results can be obtained with the frequency domain model [16, 17]. The main difference is the method of computation of the forward and backward waves from the measured waves [16]. Although net wave intensity, $\mathrm{d} I=\mathrm{d} P \mathrm{~d} U$, can be calculated straightforwardly from measured or predicted waveforms, separation into forward and backward traveling waves requires either the wave speed and blood density or the characteristic impedance. The similarity between the two methods becomes clear when $\rho c$ is regarded as an approximation of the characteristic impedance, $Z_{O}$ [10]. In that case, vessel resistance is assumed to be low compared to vessel inertance, so that longitudinal impedance can be approximated by the inertance $\rho / A$ only. Transverse impedance is related to the Young's modulus, which is a function of the square of pulse wave velocity. Previously, it was shown that similar values for forward and backward traveling wave intensities can be obtained [17] using wave intensity or frequency domain analysis. Differences between the two methods in our model simulations can be attributed to the characteristic impedance used. In case of classical wave separation, the full frequency spectrum was used for the characteristic impedance, whereas in WIA, $\rho c$ is used as an approximation of the characteristic impedance.

\subsection{Effect of aortic coarctation}

We used our previously developed model of the systemic arterial circulation [23, 27] to generate results for pressure and flow velocity waves in the reference condition, i.e. the young adult, and in case of an aortic coarctation with twofold increase in wall stiffness. Aortic coarctation causes a significant impedance mismatch between the final aortic arch segment and the stenotic thoracic aorta segment, with augmented wave reflection [13] and early return of the reflected pressure wave, leading to an increased proximal pressure. The results for the WIA confirm the existence of a strongly increased backward traveling compression wave as predicted by the conventional results of wave separation (Fig. 4). Quite similar findings were reported by Khir and Parker from measurements in dogs with aortic occlusion [10]. Earlier findings from a study in dogs [6] with artificial coarctation of the aorta also demonstrated that peak systolic aortic pressure rose strongly with progressive coarctation of the aorta, whereas a diastolic pressure rise was almost absent, although these data do not completely reproduce the DI ratio.

\subsection{Descending index}

Based on our predictions, the DI defined as the difference between the peak systolic and the valve closure pressure divided by the pulse pressure, may provide a means to estimate coarctation severity to aid in early diagnosis of the condition. Moreover, DI may be obtained noninvasively from a peripheral measurement site such as a finger artery [7] which may further confirm or discard the clinical application of our model finding.

In a paper by Wright et al. [30], the DI can be derived from published tracings of intra-arterial radial pressure measurement. From these data, we compared radial pressure waves in a subject with aortic coarctation with the 
shapes two weeks and 5 years after resection of the aorta and end-to-end anastomosis. Although the authors state that there were no differences in wave shape of the radial pressure pulse, inspection of the manuscript graphs reveals that the DI was returning to lower values after treatment. Notably, this appears to happen not immediately after surgery but several years later. Whether these data provide more than anecdotal evidence remains to be studied.

Our finding however may bear clinical relevance since mild aortic coarctation may go unnoticed in young adults for a prolonged period, and may lead to adverse remodeling of aortic wall tissue as well as the ventricular wall. Thus, identifying those individuals with moderate hypertension at risk for developing severe aortic coarctation with additional detrimental cardiovascular sequelae may prove to be a useful clinical application of the DI developed in this model study.

In contrast, in severe coarctation, a large difference in MAP between the upper and lower body part exists, with a weak femoral pulse. Since these cases often present with clinically recognizable symptoms, our findings' relevance lies with mild coarctation, which may easily remain unnoticed but may be non-invasively diagnosed from a significantly increased DI. Since the DI bears clinical relevance for cases of mild coarctation, the $80 \%$ diameter stenosis datapoint was excluded from our fit. We speculate that this index may provide a basis for further clinical study in cases of mild coarctation in the absence of overt hypertension.

\subsection{Study limitations}

Methodological limitations of our model pertain to the use of flow in the wave separation analysis and flow velocity in the WIA. The relation between flow and flow velocity is governed by the area of the vessel. In our model, flow velocity is obtained assuming negligible vessel distention. In addition, in our model it was assumed that the wave speed was pressure independent, which may contribute to the differences between wave intensity results obtained by WSA and WIA in aortic coarctation.

Additional limitations in the present model may be the absence of the influence of long-term remodeling of the vessels in response to the coarctation. Examples may be post-stenotic dilatation or outward remodeling of collateral arteries that connect the proximal and distal sites of the coarctation. Additionally the influence of physiological control mechanisms which are known to be involved in aortic coarctation were not included. An example of such a mechanism may be altered myocardial contraction [2] as resulting from ventricular hypertrophy. These mechanisms however are unlikely to alter our simulated outcomes or the agreement between the two analysis methods to a large extent. Finally, throughout the study, the same flow curve was used as input to the system. This approach seems warranted, since it was our objective to compare two methods to derive backward and forward traveling waves. Furthermore, the flow waveform is usually less influenced by the load than the pressure wave, since the heart behaves more like a flow source than a pressure source $[14,26]$. We therefore assume that using a fixed flow waveform did not introduce large errors. However, further study is needed for a detailed analysis of the effect of the flow waveform on the DI. Future study therefore may aid to derive a means of a non-invasive pressure measurement at either a finger or carotid artery that allows for early recognition of mild coarctation.

\section{Conclusion}

In the present study we have compared the application of classical wave separation in the frequency domain with the well-established technique of WIA in the time domain. Both techniques yielded similar outcomes. The results of the simulated coarctation confirm that a large backward traveling reflection wave is generated at the site of the aortic coarctation. Finally, we propose that by using the DI earlier diagnosis of aortic coarctation in young individuals presenting with mild coarctation but with absence of overt hypertension may be possible.

Acknowledgments This study was supported in part by the Netherlands Heart Foundation (grants 2006B145 and 2006B226) and by a scholarship from the Niels Stensen Foundation. We kindly acknowledge Nico Westerhof and Christina Kolyva for the critical reading of the manuscript.

Open Access This article is distributed under the terms of the Creative Commons Attribution Noncommercial License which permits any noncommercial use, distribution, and reproduction in any medium, provided the original author(s) and source are credited.

\section{References}

1. Abbruzzese PA, Aidala E (2007) Aortic coarctation: an overview. J Cardiovasc Med 8:123-128

2. Aboulhosn J, Child JS (2006) Left ventricular outflow obstruction: Subaortic stenosis, bicuspid aortic valve, supravalvar aortic stenosis, and coarctation of the aorta. Circulation 114:2412-2422. doi:10.1161/CIRCULATIONAHA.105.592089

3. Bleasdale RA, Parker KH, Jones CJ (2003) Chasing the wave. Unfashionable but important new concepts in arterial wave travel. Am J Physiol Heart Circ Physiol 284:H1879-H1885

4. Cohen M, Fuster V, Steele PM, Driscoll D, McGoon DC (1989) Coarctation of the aorta. Long-term follow-up and prediction of outcome after surgical correction. Circulation 80:840-845

5. Davies JE, Whinnett ZI, Francis DP, Manisty CH, AguadoSierra J, Willson K et al (2006) Evidence of a dominant 
backward-propagating "Suction" Wave responsible for diastolic coronary filling in humans, attenuated in left ventricular hypertrophy. Circulation 113:1768-1778. doi:10.1161/CIRCULATIONAHA. 105.603050

6. Gupta TC, Wiggers CJ (1951) Basic hemodynamic changes produced by aortic coarctation of different degrees. Circulation 3:17-31

7. Imholz BP, Wieling W, van Montfrans GA, Wesseling KH (1998) Fifteen years experience with finger arterial pressure monitoring: Assessment of the technology. Cardiovasc Res 38:605-616. doi:10.1016/S0008-6363(98)00067-4

8. Jones CJ, Sugawara M (1993) "Wavefronts" In the aortaimplications for the mechanisms of left ventricular ejection and aortic valve closure. Cardiovasc Res 27:1902-1905. doi: $10.1093 / \mathrm{cvr} / 27.11 .1902$

9. Jones CJ, Sugawara M, Davies RH, Kondoh Y, Uchida K, Parker KH, yaginuma T, Hosada S, Taylor MG, Caro CG (1994) Arterial wave intensity: Physical meaning and physiological significance. In: Recent progress in cardiovascular mechanics, vol 1. Harwood Academic, Chur, pp 129-148

10. Khir AW, Parker KH (2005) Wave intensity in the ascending aorta: Effects of arterial occlusion. J Biomech 38:647-655. doi: 10.1016/j.jbiomech.2004.05.039

11. Milnor WR (1982) Hemodynamics. Williams \& Wilkins, Baltimore

12. Murgo JP, Westerhof N, Giolma JP, Altobelli SA (1981) Manipulation of ascending aortic pressure and flow wave reflections with the valsalva maneuver: Relationship to input impedance. Circulation 63:122-132

13. O'Rourke MF, Cartmill TB (1971) Influence of aortic coarctation on pulsatile hemodynamics in the proximal aorta. Circulation 44:281-292

14. O'Rourke MF (1999) Mechanical principles. Arterial stiffness and wave reflection. Pathol Biol 47:623-633

15. Ong CM, Canter CE, Gutierrez FR, Sekarski DR, Goldring DR (1992) Increased stiffness and persistent narrowing of the aorta after successful repair of coarctation of the aorta: Relationship to left ventricular mass and blood pressure at rest and with exercise. Am Heart J 123:1594-1600. doi:10.1016/0002-8703(92)90815-D

16. Parker KH, Jones CJ, Dawson JR, Gibson DG (1988) What stops the flow of blood from the heart? Heart Vessels 4:241-245. doi: 10.1007/BF02058593

17. Parker KH, Jones CJ (1990) Forward and backward running waves in the arteries: Analysis using the method of characteristics. J Biomech Eng 112:322-326. doi:10.1115/1.2891191
18. Stergiopulos N, Young DF, Rogge TR (1992) Computer simulation of arterial flow with applications to arterial and aortic stenoses. J Biomech 25:1477-1488. doi:10.1016/0021-9290(92) 90060-E

19. Stern HC, Locher D, Wallnofer K, Weber F, Scheid KF, Emmrich P et al (1991) Noninvasive assessment of coarctation of the aorta: Comparative measurements by two-dimensional echocardiography, magnetic resonance, and angiography. Pediatr Cardiol 12:1-5. doi:10.1007/BF02238489

20. Sun YH, Anderson TJ, Parker KH, Tyberg JV (2000) Waveintensity analysis: A new approach to coronary hemodynamics. J Appl Physiol 89:1636-1644

21. Wang JJ, Parker KH (2004) Wave propagation in a model of the arterial circulation. J Biomech 37:457-470. doi:10.1016/j. jbiomech.2003.09.007

22. Westerhof BE, Guelen I, Stok WJ, Wesseling KH, Spaan JA, Westerhof $N$ et al (2007) Arterial pressure transfer characteristics: Effects of travel time. Am J Physiol Heart Circ Physiol 292:H800-H807. doi:10.1152/ajpheart.00443.2006

23. Westerhof N, Bosman F, De Vries CJ, Noordergraaf A (1969) Analog studies of the human systemic arterial tree. J Biomech 2:121-143. doi:10.1016/0021-9290(69)90024-4

24. Westerhof N, Elzinga G, Sipkema P (1971) An artificial arterial system for pumping hearts. J Appl Physiol 31:776-781

25. Westerhof N, Sipkema P, Van den Bos GC, Elzinga G (1972) Forward and backward waves in the arterial system. Cardiovasc Res 6:648-656. doi:10.1093/cvr/6.6.648

26. Westerhof N, O'Rourke MF (1995) Haemodynamic basis for the development of left ventricular failure in systolic hypertension and for its logical therapy. J Hypertens 13:943-952. doi: 10.1097/00004872-199509000-00002

27. Westerhof N, Stergiopulos N (2000) Models of the arterial tree. Stud Health Technol Inform 71:65-77

28. Westerhof N, Stergiopulos N, Noble MIM (2005) Transfer of pressure. In: Snapshots of hemodynamics. Vol 1. Springer, New York, pp 131-135

29. Westerhof N, Boer C, Lamberts RR, Sipkema P (2006) Cross-talk between cardiac muscle and coronary vasculature. Physiol Rev 86:1263-1308. doi:10.1152/physrev.00029.2005

30. Wright JL, Burchell HB, Wood EH, Hines EA Jr, Clagett OT (1956) Hemodynamic and clinical appraisal of coarctation four to seven years after resection and end-to-end anastomosis of the aorta. Circulation 14:806-814 